INPLASY

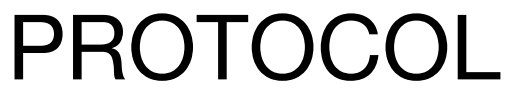

To cite: Hu et al. Circulating Level of Interleukin-6 Correlates with Poor Prognosis

in Males with COVID-19: A

Systematic Review and Metaanalysis. Inplasy protocol

2021120050. doi:

10.37766/inplasy2021.12.0050

Received: 09 December 2021

Published: 09 December 2021

Corresponding author:

Huating $\mathrm{Hu}$

1353254995@qq.com

Author Affiliation:

Macau University of Science and Technology.

Support: National Key

Research Project.

Review Stage at time of this submission: Risk of bias

assessment.

Conflicts of interest:

None declared.

\section{Circulating Level of Interleukin-6 Correlates with Poor Prognosis in Males with COVID-19: A Systematic Review and Meta-analysis}

Hu, H; Pan, H²; Li, R³ He, K; Zhang, H5; Liu, L6.

Review question / Objective: Coronavirus disease 2019 (COVID-2019), caused by severe acute respiratory syndrome coronavirus 2 (SARS-CoV-2), has become a worldwide epidemic and claimed millions of lives. Accumulating evidence suggests that males suffer more severe symptoms and higher mortality than females, but the underlying mechanism of this sex predisposition remains unclear. We aimed to explore whether inflammatory cytokines are risk factors correlated with this sex predisposition, especially in terms of the severity and mortality of COVID-19 patients. Information sources: Source: PubMed, Embase and Cochrane Library; Search dates: December 2019 to November 2021; Restriction: Language: Only English.

INPLASY registration number: This protocol was registered with the International Platform of Registered Systematic Review and Meta-Analysis Protocols (INPLASY) on 09 December 2021 and was last updated on 09 December 2021 (registration number INPLASY2021120050).

\section{INTRODUCTION}

Review question / Objective: Coronavirus disease 2019 (COVID-2019), caused by severe acute respiratory syndrome coronavirus 2 (SARS-CoV-2), has become a worldwide epidemic and claimed millions of lives. Accumulating evidence suggests that males suffer more severe symptoms and higher mortality than females, but the underlying mechanism of this sex predisposition remains unclear. We aimed to explore whether inflammatory cytokines are risk factors correlated with this sex predisposition, especially in terms of the 
severity and mortality of COVID-19 patients.

Condition being studied: Coronavirus disease 2019 (COVID-2019), caused by severe acute respiratory syndrome coronavirus 2 (SARS-CoV-2), shows a higher risk of mortality in males. According to the largest sex-disaggregated data from 47 countries, men with COVID-19 have higher morbidity than women with COVID-19 (63.8\% men; $36.2 \%$ women). In addition, the overall mortality of COVID-19 is more than 2.3 times higher in men than in women. The discrepancy in COVID-19 outcomes between male and female patients may be attributed to biological and social factors, including higher plasma levels of angiotensin-converting enzyme 2 (ACE2), transmembrane protease serine 2 (TMPRSS2), cytokine storms, smoking and age. In addition, the high rate of mortality and severe disease in male patients is also related to comorbidities, such as high blood pressure, autoimmune diseases and chronic kidney disease. However, it remains unclear which factor mainly contributes to the differences in severity and mortality between male and female patients, especially in the context of immune mechanisms.

\section{METHODS}

Participant or population: Retrospective and prospective clinical studies that enrolled patients of COVID-19 and that included at least one cytokines at baseline will be included. All studies that compared the gender with any expression levels of cytokines in Covid-19 patients will be included.

Intervention: Male patients.

Comparator: Female patients.

Study designs to be included: Both prospective and retrospective clinical study are eligible for this review.

Eligibility criteria: All the included studies met the following criteria: 1) the types of studies considered for inclusion were prospective or retrospective cohort studies comparing mild groups and severe groups; and 2) the circulating levels of IL-6 were analysed between male patients and female patients.

Information sources: Source: PubMed, Embase and Cochrane Library; Search dates: December 2019 to November 2021; Restriction: Language: Only English.

Main outcome(s): (1)Expression level of IL-6 in the male covid-19 patients and female covid-19 patients. (2)Expression level of IL-6 in the mortality group of male covid-19 patients and female covid-19 patients (3) Expression level of IL-6 in the severity group of male covid-19 patients and female covid-19 patients. The effect measure was Mean \pm SD.

Quality assessment / Risk of bias analysis: Two reviewers will independently assess risk of bias based on the following domains from recommendations from the Cochrane handbook: 1. Adequate sequence generation; 2. Allocation concealment; 3. Blinding; 4. Incomplete outcome data and how it was addressed; 5. Selective reporting of the outcome; 6. Any other biases. results of bias assessment will be presented in a figure and a graph indicating low, high or unclear risk of bias for each of the 6 items in each trial. Sensitivity analysis will be conducted based on the bias assessment to assess robustness of resultsThe methodological quality of RCTs was assessed by the Cochrane risk of bias tool. The methodological quality of retrospective studies was assessed by the modified Newcastle-Ottawascale (NOS), which consists of three factors: patient selection, comparability of the study groups, and assessment of outcome. A score of 0-9 (allocated as stars) was allocated to each study except TCTs. TCTs and observational studies achieving six or more stars were considered to be of high quality.

Strategy of data synthesis: Mean and SD will be calculated for the outcome of each expression levels of IL-6 in the groups of 
different sex. Due to expected heterogeneity among the trials, Metaanalysis using the random-effects model will be conducted.

Subgroup analysis: Subgroup analysis to determine whether the summary effects vary in relation to clinical characteristics of the trials included are pre-specified. Subgroup analyses will contain race and age (1) Race: White, African American, Asian (2) Age: $>60$ years and $\leq 60$ years Meta-regression would be used to analyzed this subgroups.

Sensitivity analysis: The main methods of sensitivity analysis are: changing the inclusion criteria (especially controversial studies), excluding low-quality studies, using different statistical methods / models to analyze the same data, etc. If there is no significant change in the results after exclusion, it indicates that the sensitivity is low and the results are more robust and reliable; On the contrary, if there are large differences or even diametrically opposite conclusions after exclusion, it indicates that the sensitivity is high and the robustness of the results is low. We should be very careful when interpreting the results and drawing conclusions, suggesting that there are important and potential bias factors related to the effect of intervention measures, and the source of dispute needs to be further clarified.

Country(ies) involved: China.

Keywords: COVID-19, cytokines, gender, mortality, severity.

Contributions of each author:

Author 1 - Huating $\mathrm{Hu}$.

Author 2 - Hudan Pan.

Author 3 - Runze Li.

Author 4 - Kancheng He.

Author 5 - Han Zhang.

Author 6 - Liang Liu. 